\title{
Listeria monocytogenes serotypes in human infections (Italy, 2000-2010)
}

\author{
Mirella Pontello(a), Anna Guaita ${ }^{(a)}$, Giuliana Sala ${ }^{(a)}$, Micaela Cipolla ${ }^{(a)}$, \\ Antonietta Gattuso $^{(\mathbf{b})}$, Michele Sonnessa ${ }^{(\mathbf{b})}$ and Monica Virginia Gianfranceschi ${ }^{(\mathfrak{b})}$ \\ (a) Dipartimento di Sanità Pubblica - Microbiologia - Virologia, \\ Università degli Studi di Milano, Milan, Italy \\ (b) Dipartimento di Sanità Pubblica Veterinaria e Sicurezza Alimentare, \\ Istituto Superiore di Sanità, Rome, Italy
}

\begin{abstract}
Background. In developed countries invasive listeriosis is an infection of great concern to public health to due its clinical severity and high fatality rate, despite its low incidence. In Europe, statistically significant increasing trends in listeriosis notification rates from 2005 to 2009 were noted in Austria, Denmark, Hungary, Italy, Spain and Sweden. Materials and methods. The standardized techniques based on phenotype to typing Listeria monocytogenes is the serotyping. In Europe, as elsewhere in the world, about $95 \%$ of L. monocytogenes strains isolated from clinical and food samples belongs to serovars $1 / 2 \mathrm{a}, 1 / 2 \mathrm{~b}, 1 / 2 \mathrm{c}$ and $4 \mathrm{~b}$. Results. The target of this work is to draw attention to this important and atypical foodborne disease, reporting epidemiological data and serotypes distribution of 251 human L. monocytogenes isolates reported during 2000-2010 to Veterinary Public Health and Food Safety Department of Istituto Superiore di Sanità, focusing on epidemiological trend of invasive listeriosis in Lombardia, a North Italian Region. The serotypes most frequently identified are $1 / 2 \mathrm{a}$, $4 \mathrm{~b}, 1 / 2 \mathrm{~b}$ (in total $92 \%$ ), but the detection of uncommon serotypes is not missing $(1 / 2 \mathrm{c}, 3 \mathrm{a}, 3 \mathrm{~b}, 4 \mathrm{~d}$ ). Conclusions. In Italy the surveillance laboratory network, as well as the foodborne disease network (ENTER-NET), has revealed in the last 11 years an increase trend of listeriosis cases reported likewise with results of Notificable National Infectious Disease surveillance System. This is probably due to a real increase of listeriosis, even if there is a greater sensitivity of the network in some regions.
\end{abstract}

Key words: Listeria monocytogenes, serotypes, surveillance.

Riassunto (Sierotipi di Listeria monocytogenes nelle infezioni umane (2000-2010). Introduzione. Nei Paesi industrializzati, nonostante la sua bassa incidenza la listeriosi invasiva è un'infezione alimentare di grande impatto per la salute pubblica, a causa della gravità delle sue manifestazioni cliniche e dell'alto tasso di letalità. In Europa, fra il 2005 e il 2009, sono stati notati incrementi, statisticamente significativi, delle notifiche di listeriosi in Austria, Danimarca, Ungheria, Italia, Spagna e Svezia. Materiali e metodi. Dal punto di vista fenotipico la tipizzazione sierologica rappresenta la tecnica più tradizionale per classificare gli stipiti di Listeria monocytogenes. In Europa come nel resto del mondo, il 95\% circa dei ceppi di L. monocytogenes isolati da campioni clinici e alimentari appartengono ai sierotipi $1 / 2 \mathrm{a}, 1 / 2 \mathrm{~b}, 1 / 2 \mathrm{c}$ e $4 \mathrm{~b}$. Risultati. L'obiettivo di questo studio è quello di attirare l'attenzione su questa grave e atipica malattia alimentare, riportando i dati epidemiologici e la distribuzione dei sierotipi, relativamente a 251 stipiti raccolti nel periodo 2000-2010, dal Dipartimento di Sanità Pubblica Veterinaria e Sicurezza Alimentare dell'Istituto Superiore di Sanità, focalizzando l'attenzione sui dati provenienti dalla Regione Lombardia. I sierotipi più frequentemente identificati sono $1 / 2 \mathrm{a}, 4 \mathrm{~b}, 1 / 2 \mathrm{~b}$ (per un complessivo $92 \%$ ) ma non è mancato il riscontro di sierotipi rari $(1 / 2 \mathrm{c}$, 3a, 3b, 4d). Conclusioni. In Italia la rete di sorveglianza di laboratorio (ENTER-NET) ha registrato negli ultimi 11 anni un incremento di casi di listeriosi dovuto a un reale incremento e a una migliore sensibilità della rete di sorveglianza in Lombardia.

Parole chiave: Listeria monocytogenes, sierotipi, sorveglianza.

\section{INTRODUCTION}

Listeria monocytogenes (L. monocytogenes) is a Gram-positive, facultative intracellular bacterial pathogen that can cause a severe invasive disease (listeriosis), mainly in immunocompromised and elderly individuals, and pregnant women, characterized by sepsis, meningitis and miscarriage [1]. In Europe, invasive listeriosis is an infection of great concern to public health due to its clinical severity (hospitalization rate $>90 \%$ ) and high fatality rate $(20 \%$ to $30 \%)$, despite its low incidence $(0.4$ cases per 100000 population), compared to salmonellosis and campylobacterios that have the highest incidence in all European countries (respectively 23.7 and 45.6 cases per 100000 population) [2]. Based on the reported fatality rates and the total numbers of reported confirmed cases, it is estimated that in 2009 there were 
approximately 270 human deaths due to listeriosis, 90 deaths due to salmonellosis and 40 deaths due to campylobacteriosis in the European Union [2]. Both sporadic and epidemic human listeriosis cases are associated with the consumption of contaminated foods [2]. Statistically significant increasing trends in listeriosis notification rates from 2005 to 2009 were noted in Austria, Denmark, Hungary, Italy, Spain and Sweden [2]. Over the last years, many reports from European countries have shown an increase of illness in older age while pregnancy-related cases is stable $[3,4]$. In the various countries, the main information on invasive listeriosis incidence come from notificable infectious diseases surveillance system. Since 1993, the reporting of listeriosis has been mandatory in Italy, and from 1996 epidemiological data are available on Ministry of Health data base (www.sanita.it). On average 38 cases/year ( 0.06 cases per 100000 population) were reported from 1996 to 2006. During 2007-2009 there was an increase of notifications with on average 105 cases/year ( 0.2 cases per 100000 population) (provisional data for 2010 confirm this trend), with the most cases are reported in the Centre-North of Italy. The national reporting system, in Italy, collects information only on gender, age and region distribution of listeriosis cases, and does not comprise sending clinical isolates to central laboratory for characterization. Besides the national reporting system, there is a voluntary laboratory-based surveillance system for the serological and molecular characterization of L. monocytogenes clinical strains. This surveillance has been included since 2009 in ENTER-NET Italia system, connected to European network ENTER-NET (Enteric Pathogen Network), involved since 2008 in the European surveillance program on Food and Waterborne Disease (FWD), coordinated by the European Centre for Disease Prevention and Control (ECDC). As priority, ECDC requires epidemiological and microbiological data about human infections due to Salmonella, VTEC, Campylobacter, $L$. monocytogenes, Shigella and Yersinia.

Human infections of L. monocytogenes are often sporadic cases and investigation on the transmission route, such as the identification of outbreaks, is complex because of the long incubation period of the invasive infection (sometimes even several weeks) and because of the probable high number of asymptomatic or paucisymptomatic infections in people exposed to the same infection vehicle $(5 \%$ of healthy carriers in the population). It follows from this that also the contemporary exposition of several people to the same food may result in little numbers of non simultaneous cases. Epidemiological survey of listeriosis needs then the support of microbiological analysis (pheno- and geno-typing) to identify cellular clones diffusion and epidemic clusters occurrence [5, 6]. Numerous discriminatory methods have been described for subtyping this organism [7]. Pulsed-field gel electrophoresis (PFGE) typing has rapidly become the standard subtyping method for investigating listeriosis outbreaks [8]. Nevertheless, for epidemiological monitoring of food and human isolates, serotyping is still required as a first level of discrimination [9]. Although 13 se- rovars are described for the species $L$. monocytogenes [10], at least $95 \%$ of the strains isolated from clinical and food samples belong to serovars $1 / 2 \mathrm{a}, 1 / 2 \mathrm{~b}, 1 / 2 \mathrm{c}$ and $4 \mathrm{~b}[2,11,12]$.

The target of this work is to draw attention to this important and atypical foodborne disease, reporting epidemiological and microbiological data collected, during 2000-2010 by Veterinary Public Health and Food Safety Department (DSPVSA) of Istituto Superiore di Sanità, focusing on epidemiological trend of invasive listeriosis in Lombardia Region. Indeed this Italian region (about 10 million people) reports to DSPVA the largest number of cases, and recently has strengthened surveillance laboratory network, combining it with reports of notificable infectious disease system called MAINF (Malattie Infettive - Infectious Diseases). We report results of L. monocytogenes isolates serotyping; further molecular analysis results for epidemiological purpose are not discussed here.

\section{MATERIALS AND METHODS Materials}

L. monocytogenes human strains and data from reports linked to the strain collected in Italy in 11 years (2000-2010).

Data and strains collection is founded on surveillance system with voluntary adhesion. These activities are referred to DSPVSA of Istituto Superiore della Sanità. Each member of this network provided several strains. The Surveillance System of Invasive Bacterical Disease, coordinated by Department of Infectious, Parasitic and Immune-Mediated Diseases of Istituto Superiore di Sanità, provided 38 strains, the surveillance system ENTER-NET Italia 7 strains and other laboratories 68 strains. In total, 138 strains have been provided by the surveillance special system of Lombardia, begun in 2006, due to the joint action of Lombardia Region and Center of Enteric Pathogens - University of Milan (CEPIS). This Italian region surveillance program is performed with the delivery of every L. monocytogenes strain isolated from hospital laboratories and compilation of an informative form. To combine results of regional and national surveillance, the form has been agreed with DSPVSA of Istituto Superiore della Sanità. Furthermore, the regional CEPIS managed the database and performed link to MAINF system.

\section{Methods \\ DSPVSA performs serological characterization of L. monocytogenes clinical isolates by Multiplex PCR [13] and sero-agglutination, referring to the serovars classification developed by Seeliger and Hohne [10]. We have built a specific database for the analysis of epidemiological and microbiological data.}

\section{RESULTS}

In the last 11 years, the DPVSA laboratory has collected 251 human strains, from blood $(61.3 \%)$ and 


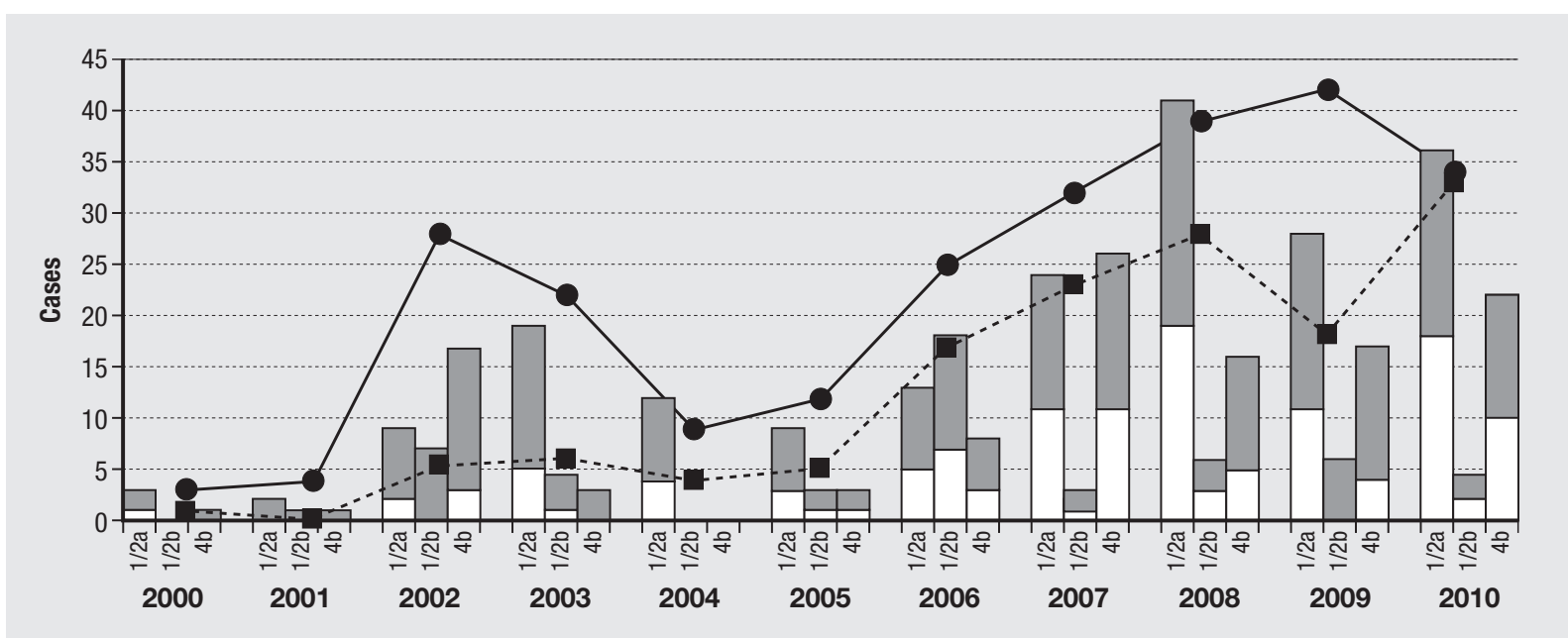

Fig. 1 | Trends of human listeriosis cases and main serotype per year (source: voluntary laboratory surveillance, 2000-2010): listeriosis cases in Italy (solid line) and in Lombardia Region (dotted line); in histogram L. monocytogenes serotypes 1/2a, 1/2b, 4b distribution in Lombardia (white bars) and in Italy (grey bars) is represented.

cerebrospinal fluid (20.7\%) referred to 243 cases of listeriosis (the strain from mother and newborn is considered one case of listeriosis). The trend of human cases of infection of L. monocytogenes reported to DPVSA is increasing in recent years, in particular between 2005 and 2009, while in 2010 there is a decrease (Figure 1). The listeriosis cases reported are mostly from northern Italy $(88.5 \%)$ and the largest part comes from Lombardia (55\%) through the special surveillance program started in 2006; specifically, in 2009 the trend in the Lombardia is decreased; while 2010 there is an increase (Figure 1).

More frequent serotypes identified are 1/2a $(46.6 \%), 4 \mathrm{~b}$ $(30.7 \%)$ and $1 / 2 b(14.7 \%)$. The trend of the 3 most frequent serotypes in Lombardia is the same of the Italian one; we remark that in 2004 no serotypes $1 / 2 b$ and $4 b$ were detected neither in Lombardia nor in the whole country (Figure 1). The identification of serotype 1/2a has increased in the latest years $(+12 \%)$, whereas the frequency of isolation of serotype $1 / 2 b$ has decreased after 2006. In last years there have been more uncommon strains (3.3\%), as 3a, 3b and 4d. In Lombardia, 55.9\% is $1 / 2 \mathrm{a}$ and $27.6 \%$ is $4 \mathrm{~b} ; 1 / 2 \mathrm{a}$ and $4 \mathrm{~b}$ are more frequent serotypes also in Emilia Romagna (23.7\% and 44.7\% respectively), the second region with higher number of infection of listeriosis in Italy (15.6\%).

Report forms were often uncompleted. Information about sex and age were provided in 218 cases $(89.7 \%)$ : the ratio between males and females is $1: 1$, with the exclusion of the first group of age, in class $0-14$ years old, in which is $2: 1$. Information about the age is given in 195 cases $(80.3 \%): 10.3 \%$ cases occurred in class under 1 years old, $6.6 \%$ in class $1-30$ years old, $27.7 \%$ in class $31-60$ years old and $55.4 \%$ in class over 61 years old. The distribution of the serotypes by age shows that $1 / 2 \mathrm{a}$ is more frequent in every age group,

Table 1 | Listeria serotypes by age and clinical data

\begin{tabular}{|c|c|c|c|c|c|c|c|c|c|}
\hline & & \multicolumn{8}{|c|}{ Serotype } \\
\hline & & $1 / 2 a$ & $1 / 2 b$ & $1 / 2 c$ & $3 a$ & $3 b$ & $4 b$ & $4 d$ & Total (\%) \\
\hline \multirow{5}{*}{ 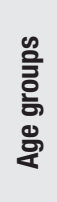 } & $<1$ & 5 & 1 & 0 & 1 & 0 & 12 & 1 & $20(10.2)$ \\
\hline & $1-30$ & 5 & 1 & 0 & 0 & 0 & 7 & 0 & $13(6.6)$ \\
\hline & $31-60$ & 27 & 10 & 1 & 0 & 0 & 14 & 2 & $54(27.7)$ \\
\hline & $>61$ & 44 & 21 & 8 & 0 & 1 & 31 & 3 & $108(55.4)$ \\
\hline & Total (\%) & $81(41.5)$ & $33(16.9)$ & $9(4.6)$ & $1(0.5)$ & $1(0.5)$ & $6(32.8)$ & $6(3.1)$ & 195 \\
\hline \multirow{5}{*}{ 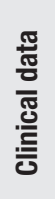 } & Pregnancy-related cases & 10 & 1 & 0 & 1 & 0 & 11 & 1 & 24 (16.5) \\
\hline & Meningitis & 19 & 8 & 0 & 0 & 0 & 12 & 1 & $40(27.6)$ \\
\hline & Septicemia & 24 & 7 & 4 & 0 & 0 & 19 & 3 & $57(39.3)$ \\
\hline & Other* & 14 & 2 & 1 & 0 & 0 & 6 & 1 & $24(16.5)$ \\
\hline & Total (\%) & $67(46.2)$ & 18 (12.4) & $5(3.4)$ & $1(0.7)$ & 0 & $48(33.1)$ & $6(4.1)$ & 145 \\
\hline
\end{tabular}


with the exception of serotype $4 \mathrm{~b}$ prevalent in class under 1 years old $(60 \%)$ ( Table 1$)$.

Clinical data are available only for 145 cases $(59.7, \%)$ : the most common clinical conditions are meningitis (40 cases) and septicemia (57 cases) in immunodepressed patients; listeriosis pregnancy-related cases are 24 cases (12 from Lombardia), mainly due to serotype 1/2a and 4b (Table 1). About cases without clinical data $33 \%$ of Lombardia cases, but $57 \%$ from other italian region), if we considered $L$. monocytogenes isolation from liquor as well as meningitis, and blood one as well as septicemia, it results that total cases are 65 and 138 respectively. Information about listeriosis outcome is available in very few cases.

\section{DISCUSSION}

The increasing trend of the cases of infection by $L$. monocytogenes in the last 11 years is probably attributable both to a real increase of listeriosis in Italy as elsewhere in Europe [2, 3] and to surveillance implementation; particularly in the last 5 years surveillance sensitivity has improved on account of laboratory-based network started in 2006 in Lombardia (1/6 of total Italian population, but about half of L. monocytogenes strains collected).

Altogether about $80 \%$ of cases is reported in the Centre-North of Italy and, even if it is possible to suspect a different incidence due to different food practice, this amount could suggest that there is an underreporting in the south of Italy especially.

Listeriosis pregnancy-related is $9.9 \%$ of total cases in Italy, while in Europe is 12\% [14]; the rate is probably underestimated because the microbiological investigation is not always performed, even in a miscarriage or premature birth, and also influenza-like illness of pregnant women is not tested for L. monocytogenes infection. In the last years the number of listeriosis pregnancy-related cases is dramatically increasing, on the contrary the percentage, indeed the trend is the same between the total cases and pregnancy-related form in 2000-2010. During this time period no seasonal trends were observed in listeriosis incidence.

The serotype distribution changes in time and in space. Comparing to previous years, from 1990 to 1999 [11], in the last eleven years there is an increasing of cases due to $1 / 2 \mathrm{a}$ and a decrease of the cases due to $4 \mathrm{~b}$ serotype. This change is reported also from other European countries like Finland, Great Britain, Switzerland, Denmark and Sweden, in addition to extra-European countries [12]. The serotype shift can be related to blood stream infection, that is becoming a more common clinical presentation than meningitis, and where serotypes $1 / 2 \mathrm{a}$ is more frequently isolated than serotype $4 \mathrm{~b}$ (Table 1). The rate of isolation of serotype $4 \mathrm{~b}$ is higher among patients suffering from meningoencephalitis than in patients suffering from blood stream infection, indicating that strains of serotype $4 \mathrm{~b}$ may be more virulent in so far as is more able to pass the blood-brain barrier.
More patients with predisposing conditions seem to suffer from blood stream infection due to L. monocytogenes than meningoencephalitis. The reason for this finding may be that the bacteremia is the first step towards the bloodstream to reach the central nervous system or nowadays use of blood culture is more frequent in cases of "hyperthermia patient" with more opportunity to capture the primary stage of infection. A likely explanation is that patients with predisposing conditions are in closer contact with the health system and, therefore, are more likely to have drawn blood for culture in case of fever than a person with no predisposing condition [15].

In north Italy the prevalent serotype is $1 / 2 \mathrm{a}(47 \%)$, while in the centre is $4 \mathrm{~b}(44 \%)$; but the number of the collected cases is so different that not comparison between the geographical areas is achievable.

In recent years (2006, 2009 and 2010), in Italy, eight cases of listeriosis have been associated with uncommon serotypes ( $3 \mathrm{a}, 3 \mathrm{~b}$ and $4 \mathrm{~d}$ ), with two cases pregnancy related (Table 1). To date, in Europe as elsewhere in the world, no case linked to these uncommon serotypes has been reported, with the only exception for serotype $3 \mathrm{a}$, which is also reported from Finland where caused an serious outbreak transmitted by an unusual vehicle (pasteurized butter) involving 25 patients and determining a very high fatality rate (about 40\%) [16]. Further analysis will be needed to explain the emergency of these uncommon serotypes, including food microbiological analysis to verify in which kind of food these serotypes are present, and genotyping analysis of strains to verify likely change in virulence.

The unsatisfactory degree of completeness in the compilation of the laboratory report forms is stressed from the data analysis and is attributable to a bad communication between clinicians and microbiologists; moreover, the laboratory collection can not load of clinical data because the database would be very complex and repetitive, indeed some epidemiological data are also reported in the report forms from notificable infectious diseases surveillance system. A statistical analysis performed linking two databases (from notificable infectious disease system and laboratory network) is more simple and useful to carry out an integrated surveillance system. In Lombardia Region an epidemiological integrated surveillance has begun and the same procedure can be extended to other Italian geographical areas and considered for other infectious diseases especially about food-borne diseases.

\section{CONCLUSIONS}

In Italy the surveillance laboratory networtk, as well as the foodborne disease network (ENTERNET), has revealed in the last 11 years an increase trend of listeriosis cases reported likewise with results of Notificable National Infectious Disease Surveillance System. This is probably due to a real increase of listeriosis, even if there is a greater sen- 
sitivity of the network in some regions. Listeriosis is a serious and preventable foodborne infection for which vulnerable populations and high risk foods have been known since many decades. While surveillance can be further enhanced to develop food safety policy into the future, it is important the well established vulnerable populations are protected as soon as possible. Education of consumers, especially those who fall into the high risk groups, about highrisk foods will be necessary to keep morbidity and mortality due to listeriosis as low as possible.

An efficient surveillance system is a responsibility shared by many patterns in the epidemiologic and public health services, in microbiological laboratories, both in medical and veterinary fields. Close collaboration between general physicians, microbiologists and epidemiologists is required to collect complete information about listeriosis, including outcome.

\section{References}

1. Allerberger F, Wagner M. Listeriosis: a resurgent foodborne infection. Clin Microbiol Infect 2010;16:16-23. http://dx.doi.org/10.1111/j.1469-0691.2009.03109.x

2. European Food Safety Authority/European Centre for Disease Prevention and Control (EFSA/ECDC). The European Union summary report on trends and sources of zoonoses, zoonotic agents and food-borne outbreaks in 2009. EFSA Journal 2011;9(3):2090. http://dx.doi.org/10.2903/j.efsa.2011.2090

3. Gianfranceschi MV, Gattuso A, D’Ottavio MC, Fokas S, Aureli P. Results of a 12-month long enhanced surveillance of listeriosis in Italy. Eurosurveillance 2007;12:7-8.

4. Goulet V, Hedberg C, Le Monnier A, de Valk H, Increasing incidence of listeriosis in France and other European countries. Emerg Infect Dis 2008;14:734-40. http://dx.doi.org/10.3201/eid1405.071395

5. Den Bakker HC, Fortes ED, Wiedmann M. Multilocus sequence typing of outbreak-associated Listeria monocytogenes isolates to identify epidemic clones. Foodborne Path Dis 2010;7:257-65.

http://dx.doi.org/10.1089/fpd.2009.0342

6. Mammina C, Aleo A, Romani C, Pellisier N, Nicoletti P, Pecile P, Nastasi A, Pontello M. Characterization of Listeria monocytogenes isolates from human listeriosis cases, Italy. J Clin Microbiol 2009;47:2925-30. http://dx.doi.org/10.1128/JCM.00102-09

7. Graves LM, Swaminathan B, Hunter SB. Subtyping Listeria monocytogenes. In: Ryser ET, Marth EH (Ed). Listeria, listeriosis and food safety. New York: Dekker; 2007. p. 279-97. http://dx.doi.org/10.1201/9781420015188.ch9

8. Graves LM, Swaminathan B. PulseNet standardized protocol for subtyping Listeria monocytogenes by macrorestriction and pulsed-field gel electrophoresis. Int J Food Microbiol 2001;65:55-62.

http://dx.doi.org/10.1016/S0168-1605(00)00501-8
This surveillance system, in spite of the voluntary reports of L. monocytogenes, will enrich the epidemiological knowledge of listeriosis in Italy, through joint analysis of epidemiological and microbiological data. Also data presented in this study show the importance of voluntary reports, that allow to collect information, like clinical aspects, predisposing conditions, including pregnancy, and serotypes. All these elements, that are not collected through the national reporting system, are very important to give information to ECDC that requires these set of data to estimate the burden of this important foodborne disease.

\section{Conflict of interest statement}

There are no potential conflicts of interest or any financial or personal relationships with other people or organizations that could inappropriately bias conduct and findings of this study.

Received on 2 August 2011.

Accepted on 12 January 2012.

9. Kérouanton A, Marault M, Petit L, Grout J, Tam Dao T, Brisabois A. Evaluation of a multiplex PCR assay as an alternative method for Listeria monocytogenes serotyping. J Microbiol Meth 2010;80:134-7.

http://dx.doi.org/10.1016/j.mimet.2009.11.008

10. Seeliger HPR, Hohne K. Serotyping of Listeria monocytogenes and related species. Meth Microbiol 1979;13:31-49. http://dx.doi.org/10.1016/S0580-9517(08)70372-6

11. Gianfranceschi MV, Gattuso A, Tartaro S, Aureli P. Incidence of Listeria monocytogenes in food and environmental samples in Italy between 1990 and 1999: serotype distribution in food, environmental and clinical samples. Eur J Epidemiol 2003;18:1001-6. http://dx.doi.org/10.1023/A:1025849532417

12. Gianfranceschi MV, D’Ottavio MC, Gattuso A, Bella A, Aureli P. Distribution of serotypes and pulsotypes of Listeria monocytogenes from human, food and environmental isolates (Italy, 2002-2005). Food Microbiol 2009;26:520-6. http://dx.doi.org/10.1016/j.fm.2009.03.003

13. Doumith M, Buchrieser C, Glaser P, Jacquet C, Martin P. Differentiation of the Major Listeria monocytogenes Serovars by Multiplex PCR. J Clin Microbiol 2004;42:3819-22. http://dx.doi.org/10.1128/JCM.42.8.3819-3822.2004

14. Mook P, Grant K A, Little C L, Kafatos G, Gillepsie A. Emergence of pregnancy-related listeriosis amongst ethnic minorities in England and Wales. Eurosurveillance 2010;15:17-23.

15. Swaminathan B, Gener-Smidt P. The epidemiology of human listeriosis. Microb Infect 2007;9(10):1236-43. http://dx.doi.org/10.1016/j.micinf.2007.05.011

16. Lyytikainen O, Autio T, Maijala R, Ruutu P, HonkanenBuzalski T, Miettinen M, Hatakka M, Mikkola J, Anttila VJ, Johanssson T, Rantala L, Aalto T, Korkeala H, Siitonen A. An outbreak of Listeria monocytogenes serotype 3 a infections from butter in Finland. J Infect Dis 2000;181(5);1838-41. http://dx.doi.org/10.1086/315453 